\title{
PREVALÊNCIA DA ANEMIA EM CRIANÇAS AVALIADA PELA PALIDEZ PALMAR E EXAME LABORATORIAL: IMPLICAÇÕES PARA ENFERMAGEM
}

Prevalence of anemia in children assessed by clinical method known as "palmar pallor" and the laboratory exam: implications for nursing

Sentimientos de mujeres-madres delante de la cirugía neonatal en las malformaciones congénitas

Ethel Bastos da Silva ${ }^{1}$

Melânia Sartori Villani

Alice do Carmo Jahn ${ }^{3}$

Marta $\mathrm{Coco}^{4}$

\section{RESUMO}

Buscou-se analisar a prevalência de anemia ferropriva em crianças e comparar os dados obtidos pelo método clínico "palidez palmar" e exame laboratorial em uma Estratégia de Saúde da Família no município de Panambi/RS. Pesquisa exploratória/descritiva, quantitativa, realizada de março a junho de 2009. Foram sujeitos 41 crianças de 2 meses a 5 anos. Os dados foram analisados pela estatística descritiva. Das crianças, 51,2\% eram do sexo masculino; 41,46\% estavam na faixa etária de 0 a 12 meses; $53,6 \%$ das famílias situavam-se no nível social D e 43,9\% dos chefes de família tinham o Ensino Fundamental completo ou o Ensino Médio incompleto. Pela palidez palmar, a prevalência de anemia foi de $51,2 \%$ e pelo exame laboratorial, de $58,53 \%$. 0 estudo mostra que a palidez palmar e o exame laboratorial, quando usados juntos, fornecem um diagnóstico mais preciso da anemia ferropriva, podendo ser inseridos na prática assistencial da enfermagem na ESF.

Palavras-chave: Enfermagem. Cuidado da Criança. Anemia Ferropriva. Palidez. Diagnóstico.

\begin{abstract}
We sought to examine the prevalence of iron deficiency anemia in children and compare the data obtained by the clinical method known as "palmar pallor" and the laboratory exam on the Family Health Strategy services in the municipality of Panambi-RS. The exploratory, descriptive and quantitative research was carried out from March to June 2009. The subjects were 41 children from the age of 2 months up to 5 years old. The data were analyzed by descriptive statistics. Out of the total number of children, $51,2 \%$ of them were male; $41,46 \%$ of them fit in the age frame from 0 to 12 months old; $53,6 \%$ belonged to families of $D$ social level while $43,9 \%$ of the family chiefs have complete Elementary Education or incomplete Junior High School. Considering the palmar pallor, the prevalence of anemia was $51,2 \%$ and as per the lab exam, by $58,53 \%$. The study shows that the palmar pallor and the laboratory exam when used together provide a more accurate diagnosis of iron deficiency anemia, can be inserted in the professional nursing practice in the ESF.
\end{abstract}

Key words: Nursing. Child Care. Anemia Iron-Deficieny. Pallor. Diagnosis.

\section{Resumen}

Se intenta analizar la prevalencia de anemia por deficiencia de fierro en niños y comparar el método clínico "palidez palmar" y el examen de laboratorio en una Estrategia de Salud de la Familia en la municipalidad de Panambi-RS. La pesquisa exploratoria, descriptiva y cuantitativa fue realizada de marzo hasta junio de 2009. Los sujetos fueron 41 niños de 2 meses a 5 años de edad. Los datos fueron analizados por la estadística descriptiva. Del total de niños, 51,2\% eran del sexo masculino; $41,46 \%$ estaban en la franja etaria de 0 a 12 meses; 53,6\% de las familias se sitúan en el nivel social D y $43,9 \%$ de los jefes de familia tienen la Enseñanza Fundamental Completa o la Enseñanza Media Incompleta. Por la palidez palmar, la prevalencia de anemia fue de $51,2 \%$ y por el examen de laboratorio, de $58,53 \%$. El estudio muestra que la palidez palmar y el examen de laboratorio, cuando usados juntos, proveen un diagnóstico más preciso de la anemia por deficiencia de fierro, pudiendo ser inserido en la práctica asistencial de enfermería en la ESF.

Palabras Clave: Enfermería. Cuidado del Niño. Anemia Ferropénica. Palidez. Diagnóstico.

\footnotetext{
'Mestre em Enfermagem pela Universidade Federal de Santa Catarina. Professora Assistente do Departamento de Enfermagem da Universidade Federal de Santa Maria/Centro de Educação Superior Norte do Estado. Palmeira das Missões-RS. Brasil. Email: ethelbastos@hotmail.com; ${ }^{2}$ Enfermeira Graduada pela Universidade de Cruz Alta. Cruz Alta-RS. Brasil. Email: melvillani@hotmail.com; ${ }^{3}$ Mestre em Enfermagem Fundamental pela Escola de Enfermagem de Ribeirão Preto- USP. Professora Assistente do Departamento de Enfermagem da Universidade Federal de Santa Maria/Centro de Educação Superior Norte do Estado. Palmeira das Missões-RS. Brasil. Email: alicejahn@terra.com.br; ${ }^{4}$ Mestre em Enfermagem pela Escola de Enfermagem da Universidade Federal do Rio Grande do Sul. Professora Assistente do Departamento de Enfermagem da Universidade Federal de Santa Maria/Centro de Educação Superior Norte do Estado. Palmeira das Missões-RS. Brasil. Email: martafwcocco@yahoo.com.br
} 


\section{INTRODUÇÃO}

As doenças infecciosas e as deficiências nutricionais têm sido responsáveis pela morte de 11 milhões de crianças por ano no mundo, ${ }^{1}$ segundo a Organização Mundial de Saúde (OMS). Estima-se que, no mundo todo, $47,4 \%$ das crianças menores de 5 anos tenham anemia, com valores distribuídos em 3,4\% na América do Norte a 64,6\% na África. Na América Latina, os valores são de 39,5\% para crianças menores de 5 anos e $31,1 \%$ para mulheres grávidas ${ }^{2}$.

Já no Brasil, os índices também são alarmantes, especialmente em crianças menores de 2 anos, sendo que a extensão de anêmicos é de $50 \%$ a $83 \%^{3}$, variando de acordo com as regiões, grupos populacionais infantis e exposição a fatores de risco. No Rio Grande do Sul, em Pelotas, os fatores encontrados foram menor idade da criança, cor não branca, presença de família numerosa e classe social $E,{ }^{4}$ já na cidade de São Paulo, foram menor idade e idade dos responsáveis da criança menor de 25 anos $^{5}$. Na cidade de Fortaleza-Ceará, os fatores que apresentaram significância foram a idade e 0 estado nutricional ${ }^{6}$.

Os estudos acima relatados avaliaram a anemia com exame de sangue pelo nível de hemoglobina e confirmam a anemia como um problema relevante na infância, que impede a criança de crescer e desenvolver-se de forma saudável de acordo com o esperado para a faixa etária. A anemia causada pela deficiência de ferro proporciona perdas relacionadas ao desenvolvimento motor, cognitivo, mental e também predisposição para ocorrência de infecções. Ocorre diminuição da produtividade, rendimento corporal e dificuldades com aprendizado. ${ }^{2,7}$

Os autores reforçam que a anemia ferropriva é decorrente de múltiplos fatores associados, os quais, quando conhecidos, podem ser sanados por meio de ações de orientação e acompanhamento das famílias de crianças expostas a essas condições ${ }^{4-5-6}$.

As condições socioeconômicas e demográficas das populações infantis influenciam no aparecimento da anemia ferropriva, sendo fundamental a realização de estudos diagnósticos para que as medidas de controle e prevenção sejam implementadas. Nesse enfoque, a Organização Mundial da Saúde (OMS), juntamente com o Fundo das Nações Unidas para Infância e Juventude (UNICEF), na década de 90, criaram a Estratégia de Atenção Integrada às Doenças Prevalentes na Infância (AIDPI), com o objetivo de diminuir as altas taxas de mortalidade e morbidade infantil em todo o mundo ${ }^{8} \mathrm{No}$ Brasil, a AIDPI foi adaptada às características das crianças de acordo com a realidade local, e é executada principalmente pelas Estratégias de Saúde da Família (ESF). ${ }^{9}$

De acordo com as orientações da AIDPI, para realizar avaliação da anemia, é necessário utilizar o sinal clínico da "palidez palmar", que avalia a coloração rósea avermelhada da pele da palma da mão da criança em comparação com a cor da palma da mão da mãe. Todos os resultados obtidos na consulta são anotados em um formulário de registro específico, com o objetivo principal de realizar uma triagem rápida e identificar precocemente diversas doenças, fazendo com que elas possam ser tratadas e curadas em um curto período de tempo. ${ }^{1-9-10}$

Os procedimentos prestados pela AIDPI congregam todas as orientações do Ministério da Saúde relacionadas à promoção, prevenção e tratamento das doenças. No que se refere à promoção e proteção, priorizam o acompanhamento do crescimento e desenvolvimento, imunizações, aleitamento materno e orientações nutricionais. No que se refere à prevenção e tratamento das doenças infantis mais frequentes, procuram 0 controle dos agravos à saúde, tais como: desnutrição, doenças diarreicas, infecções respiratórias agudas, doenças febris entre outros. ${ }^{9}$

Os estudos realizados pelo método "palidez palmar" têm trazido contribuições significativas quanto ao método e aconselham o uso, mas recomendam prudência e aprimoramento da técnica por parte dos avaliadores. ${ }^{11}$ Salienta-se que esse método é recomendado para populações em que a anemia é grave, pois quanto maior o grau de anemia, mais sinais clínicos a criança apresenta. ${ }^{12}$

Uma vez que a ESF presta atendimento a crianças, parcela vulnerável da população a desenvolver a anemia por deficiência de ferro, e sendo a anemia um grave problema de saúde pública e que afeta uma grande parte da população infantil, o estudo objetivou analisar a prevalência de anemia ferropriva em crianças de 2 meses a 5 anos de idade atendidas em uma ESF no município de Panambi/RS e comparar os dados de prevalência obtidos pelos métodos de avaliação da palidez palmar e nível de hemoglobina no sangue.

\section{MÉTODO}

Esta é uma pesquisa exploratória-descritiva com abordagem quantitativa. 0 campo do estudo foi uma unidade de ESF do município de Panambi, localizado na região noroeste do estado do Rio Grande do Sul, sendo o período de realização da coleta dos dados de março a junho de 2009.

A população do estudo foi composta por crianças de 2 meses a 5 anos, que buscaram atendimento na ESF no período selecionado e cujas mães aceitaram que a criança participasse do estudo. Os critérios de exclusão foram crianças que apresentassem alguma doença que comprometesse a integridade da pele, doenças reumáticas, nefropatias, e também sintomas como febre, diarreia e vômito, com diagnóstico confirmado no momento da consulta. Assim a amostra constituiu-se de 41 crianças.

As crianças foram avaliadas por meio do método proposto pela AIDPI, que indica a observação da palma da mão da criança e da palma da mão da mãe no momento da consulta pediátrica. Conforme preconizam as normas do AIDPI, a avaliação clínica da palidez palmar foi feita de acordo com 
experiência, julgamento e consenso de dois examinadores acerca da alteração da coloração róseo-avermelhada da pele, considerada como normal. ${ }^{8}$ Utilizou-se ambiente claro, com luz natural ou artificial com lâmpada fluorescente. Após a classificação as mãe eram encaminhadas para realizarem exames laboratoriais em seus filhos para confirmar a avaliação.

A validação do diagnóstico clínico foi realizada por meio da avaliação da hemoglobina circulante através de um hemograma solicitado pelo médico pediatra após a avaliação palmar. Para os estágios de gravidade da anemia, também foi adotado o critério da OMS (Hb d"7g/dl - anemia grave, Hb entre 7 e 9,9g/dl - anemia moderada, $\mathrm{Hb}>10 \mathrm{~g} / \mathrm{dl}$-anemia leve). ${ }^{7}$

Para identificação dos fatores de risco para anemia na população em estudo, foi aplicado um questionário elaborado pela própria pesquisadora com variáveis relacionadas à criança tais como sexo, faixa etária, renda familiar e grau de instrução do chefe da família. Para a avaliação socioeconômica, foi utilizado o Critério de Classificação Econômica Brasil 2008 (CCEB), determinado pela Associação Brasileira de Empresas de Pesquisa (ABEP), que estima o poder de compra das pessoas e famílias através da posse de itens de consumo (televisão em cores, geladeira, freezer, máquina de lavar, vídeo-cassete, automóvel, empregada mensalista e banheiro) e grau de escolaridade do chefe da família. A classe econômica é estratificada em sete categorias, com a respectiva renda média familiar, baseada no salário mínimo (SM) vigente.
Os dados analisados por meio da estatística descritiva do Programa Excel 2003, considerando as porcentagens e frequência absoluta e relativa, serão apresentados em forma de tabelas. A seguir, foram discutidos e descritos de acordo com a literatura pertinente.

A pesquisa obedeceu à Resolução 196/96 do Conselho Nacional de Saúde e foi aprovada pelo Comitê de Ética em Pesquisa da Universidade de Cruz Alta, CEP/ UNICRUZ, sob parecer consubstanciado 097/08. Obtiveramse a autorização por escrita do Secretário da Saúde e a assinatura do Termo de Consentimento Livre e Esclarecido dos responsáveis pelas crianças.

\section{RESULTADOS E DISCUSSÃO}

\section{Caracterização das crianças de 2 meses a 5 anos atendidas na ESF em um município da região noroeste do Rio Grande do Sul}

As características das crianças avaliadas pelo método clínico palidez palmar e exame laboratorial no que se refere ao sexo, faixa etária e classificação econômica das famílias e grau de instrução dos chefes podem ser fatores determinantes no aparecimento da anemia e estão dispostas nas Tabelas 1 e 2, com as discussões fundamentadas em estudos brasileiros.

Tabela 1 Caracterização das crianças de 2 meses a 5 anos de acordo com a faixa etária e sexo atendidas em uma ESF. Panambi/RS, 2009.

\begin{tabular}{lll}
\hline Variáveis & $\mathrm{N}=41$ & $\%$ \\
\hline Sexo & & \\
Masculino & 21 & 51,2 \\
Feminino & 20 & 48,7 \\
Faixa Etária (meses) & & \\
2-12 meses & 17 & 41,4 \\
13-24 meses & 10 & 24,3 \\
25-36 meses & 03 & 7,31 \\
37-60 meses & 11 & 26,8 \\
\hline
\end{tabular}

Quanto à caracterização das crianças, a pesquisa revela que $51,2 \%$ eram do sexo masculino e $48,7 \%$, do sexo feminino. Achados semelhantes fundamentam o presente estudo, como o realizado em creches de Belo Horizonte/Minas Gerais, cujo objetivo foi avaliar o estado nutricional e prevalência de anemia em crianças e que encontrou $51,7 \%$ de crianças do sexo masculino, assim como outro realizado em Fortaleza- Ceará cujo objetivo foi identificar fatores de risco para a anemia em crianças que frequentavam creches, e que também verificou maior frequência de crianças do sexo masculino, com um percentual de $53,9 \% .^{13-6}$

Quanto à faixa etária, $41,4 \%$ das crianças estavam entre 0 a 12 meses e $26,8 \%$ estavam na faixa de 37 a 60 meses, o que indica que, além de a anemia estar aumentando de forma alarmante nos últimos anos, está se instalando muito precocemente, colaborando para o aumento dos índices de mortalidade infantil no mundo todo, ${ }^{3}$ conforme apontam dados do estudo realizado em Pelotas - RS em que a prevalência de anemia em menores de 12 meses de idade foi de $81,1 \%$, decrescendo para $77,5 \%$ em crianças de 12 a 23, 9 meses. $^{4}$

Cabe salientar que: "a maior frequência na deficiência de ferro ocorre entre os 6 a 24 meses de idade-período de acelerado crescimento do cérebro e desdobramento e fundamentação dos processos mentais e motores" " 3:358 Nesse sentido, mais uma vez se recomenda o fortalecimento de ações multidisciplinares de 
saúde com enfoque na puericultura e reconhece-se o espaço privilegiado da estratégia de saúde da família como local de intervenção. 0 acompanhamento do crescimento e desenvolvimento da criança é, sem dúvida, uma das ações de promoção da saúde integral, que quando bem realizada, pode identificar sinais que podem comprometer o pleno desenvolvimento biológico, cognitivo e sensorial da criança.

Na sequência, a Tabela 2 apresenta a classificação econômica da família e o grau de instrução dos chefes das famílias das crianças atendidas na ESF.

Tabela 2 Caracterização da classificação econômica e grau de instrução do chefe da família de crianças de 2 meses a 5 anos atendidas em uma ESF . Panambi/RS, 2009.

\begin{tabular}{|c|c|c|}
\hline Variáveis & $\mathrm{N}=41$ & $\%$ \\
\hline \multicolumn{3}{|l|}{ Classficação Econômica } \\
\hline B2 & 2 & 4,8 \\
\hline C & 12 & 29,3 \\
\hline D & 21 & 51,2 \\
\hline E & 5 & 12,1 \\
\hline \multicolumn{3}{|l|}{ Grau de Instrução do Chefe da Familia } \\
\hline Analfabetos e ou $4^{\mathrm{a}}$ séne do Ensino Fundamental incompleto & 9 & 21,9 \\
\hline $4^{\text {a }}$ série ou até a $8^{\mathrm{a}}$ série do Ensino Fundamental incompleto & 14 & 34,1 \\
\hline Ensino Fundamental completo ou o Ensino Médio incompleto & 18 & 43,9 \\
\hline
\end{tabular}

Os dados socioeconômicos foram coletados e anotados através de informação verbal das mães pelo Critério de Classificação Econômica do Brasil, feito pela Associação Brasileira de Empresas de Pesquisa (ABEP). Nesse instrumento, o nível socioeconômico é expresso em renda familiar mensal. Constatou-se que $4,8 \%$ das famílias recebem, em média, $\mathrm{R} \$ 1.659,00$ (mil seiscentos e cinquenta reais) por mês, portanto classificadas no nível B2; 29,3\% recebem em torno de $R \$ 927,00$ (novecentos e vinte e sete reais) por mês, classificadas no nível C; $51,2 \%$ das famílias classificam-se no nível $D$ e recebem em média $\mathrm{R} \$ 424,00$ (quatrocentos e vinte e quatro reais) por mês; e $12,1 \%$ das famílias recebem em média $R \$ 207,00$ (duzentos e sete reais) por mês, classificadas em nível $\mathrm{E}$.

0 nível econômico das famílias pode influenciar no aparecimento da anemia em crianças. Essa afirmação é comprovada em um estudo onde se observou maior prevalência de anemia na classe social E em comparação com a classe social D. ${ }^{4}$ Quanto menor a renda da família, maiores são as chances de uma criança se encontrar anêmica. As comunidades que residem em zonas rurais ou na periferia das cidades recebem baixos salários, apresentam baixos níveis educacionais, especialmente quanto à escolaridade paterna, implicando na baixa disponibilidade de recursos para suprir as necessidades essenciais à qualidade de vida e acesso mínimo à alimentação. ${ }^{14}$ Essas características são semelhantes às da população deste estudo e podem ter influenciado na prevalência da anemia ferropriva das crianças, e, quando associadas a outros fatores, podem ter contribuído para a instalação e manutenção da anemia em parte das crianças.

Embora a prevalência de anemia seja significativamente maior na população com nível socioeconômico mais baixo, um estudo realizado na Bahia mostra que os indivíduos com estrato econômico mais privilegiado também apresentam ocorrências relativamente altas de anemia. ${ }^{15}$

Essa constatação pode ser atribuída a fatores que não interferem nas condições de classe e atingem os indivíduos, independentemente do nível socioeconômico, e que podem prejudicar tanto o consumo dos alimentos ricos em ferro quanto o consumo dos alimentos que favorecem sua absorção. Isso porque muitas crianças, mesmo com condições de renda alta, podem ter uma dieta ineficaz, com alimentos pobres em ferro. ${ }^{15}$

Conforme o grau de instrução, a pesquisa mostrou que $21,9 \%$ dos chefes de família eram analfabetos ou estudaram até a $4^{\mathrm{a}}$ série do Ensino Fundamental, incompleto, 34,1\% fizeram a $4^{a}$ série ou até a 8 a série do Ensino Fundamental, incompleto e 43,9\% tinham o Ensino Fundamental completo ou o Ensino Médio incompleto. Não foi encontrado nenhum chefe de família que tenha realizado o curso superior, completo ou incompleto.

A população estudada apresenta dados estatísticos que comprovam acesso à informação através dos meios de comunicação pelos itens de consumo adquiridos como o rádio e a televisão. Estes veículos de informações, no entanto, nem sempre têm influência sobre as práticas de cuidado das famílias. As informações repassadas pelos profissionais das unidades de saúde podem modificar hábitos de vida das populações. Nesse sentido, quando pais e ou familiares recebem suporte dos profissionais de saúde, às vezes, alteram o cuidado prestado à criança melhorando a qualidade de vida e diminuindo a exposição a situações de risco. ${ }^{16}$

Um estudo realizado na cidade de Uberlândia - MG em uma unidade de saúde com 70 crianças revelou a baixa escolaridade materna e reduzida renda mensal, caracterizando a população como carente e, portanto, vulnerável à instalação de anemia. ${ }^{17}$ Cabe referir que o grau de instrução da mãe está diretamente relacionado ao cuidado à criança, ou seja, quanto 
maior a escolaridade, melhores serão as condições de cuidado ${ }^{15}$. Ressalta-se que a escolaridade da mãe pode ser um dos fatores determinantes da anemia, e não o único, e neste estudo não foi identificado o grau de escolaridade materna, somente o grau de instrução do chefe da familia que, associado a outros fatores, pode potencializar o aparecimento da anemia em crianças.

0 grau de instrução dos pais pode ser considerado um importante fator socioeconômico na determinação da anemia, levando em consideração que a maior escolaridade oferece uma maior chance de emprego, com um salário melhor, proporcionando dessa maneira maiores condições de adquirir alimentos saudáveis e ricos em ferro. ${ }^{15}$

Os dados do presente estudo confirmam que as condições sociodemográficas, como sexo, idade, situação econômica e o grau de instrução, são fatores que interferem diretamente na incidência da anemia ferropriva em crianças, sendo que esses últimos dois aspectos, quando reconhecidos pelas equipes de saúde e setores de assistência social, podem ser alterados e determinar menores coeficientes da doença.

Os fatores socioeconômicos são considerados constituintes do eixo social da vulnerabilidade de crianças e suas familias, e, nesse aspecto, cabe referir, que quando a equipe de saúde considera as vulnerabilidades em que crianças e suas famílias estão imersas, pode delinear e realizar um cuidado integral à saúde da criança, especialmente no que se refere a orientaç̃̃es oferecidas quanto ao diagnóstico e tratamento de doenças, uma vez identificada a dificuldade de compreensão por parte dos pais e ou cuidadores. ${ }^{16}$

\section{Prevalência da Anemia Ferropriva pelo Método Palidez Palmar x Exame Laboratorial}

No Brasil, os artigos publicados no período de 11 anos relacionados à anemia ferropriva em crianças menores de dois anos constataram que a prevalência é de $53 \%$, confirmando valores estimados pela Organização Mundial de Saúde (OMS), considerados elevados. No entanto, recomenda-se a necessidade de mais estudos com amostras de base populacional, ${ }^{18}$ pois o tipo de anemia mais encontrado é 0 leve. $^{12}$

Tradicionalmente, a anemia é mensurada pelo exame laboratorial. 0 nível de hemoglobina é considerado o meio mais sensível para detectar a anemia. A OMS determina a anemia grave pela $\mathrm{Hb} \mathrm{d}$ " $\mathrm{g} / \mathrm{dl}$, anemia moderada pela $\mathrm{Hb}$ entre 7 e $9,9 \mathrm{~g} / \mathrm{dl}$ e anemia leve pela $\mathrm{Hb}<10 \mathrm{~g} / \mathrm{dl} .{ }^{7} 0$ ponto de corte para classificação da anemia em crianças desse estudo foi determinado pela orientação da OMS: 10 mg de hemoglobina para crianças de 2 meses a 5 anos de idade.

0 método do sinal clínico da palidez palmar tem sido disseminado nos países em desenvolvimento e adotado em alguns municípios do Brasil pela implantação da estratégia AIDPI. De acordo com a OMS, o exame clínico de palidez cutânea pode ser adotado para detectar anemia grave. ${ }^{8-9}$ Essa orientação leva a questionamentos quanto à sensibilidade do método para avaliação da anemia leve e moderada.

A Tabela 3 revela a prevalência da anemia pelo método palidez palmar e exame laboratorial em crianças atendidas em uma unidade de saúde da família de um município da região noroeste do Rio Grande do Sul em 2009.

Tabela 3. Distribuição da prevalência de anemia ferropriva pelo método palidez palmar e exame laboratorial em crianças de 2 meses a 5 anos usuárias de uma ESF de Panambi, 2009.

\begin{tabular}{|c|c|c|}
\hline Variáveis & $\mathrm{N}$ & $\%$ \\
\hline \multicolumn{3}{|c|}{ Prevalência da Anemia pelo Método Palidez Palmar } \\
\hline Palidez palmar leve, moderada & 20 & 48,8 \\
\hline Palidez grave & 1 & 2,4 \\
\hline Pele normal (ausência de anemia) & 20 & 48,8 \\
\hline \multicolumn{3}{|c|}{ Prevalência da Anemia pelo Hemograma } \\
\hline Anemia leve (7 e 9,9 g/dl) ; & 12 & 50,0 \\
\hline Anemia Moderada (<10 g/dl); & 11 & 45,8 \\
\hline Anemia Grave (? $7 \mathrm{~g} / \mathrm{dl}$ ) & 1 & 4,1 \\
\hline
\end{tabular}

A Tabela 3 mostra que, pelo exame clínico da palidez palmar, foi identificado o grau de anemia ferropriva leve e moderada com $48,8 \%$, e 2,4\% anemia grave. A prevalência total de anemia foi de $51,2 \%$. A partir dos resultados dos exames laboratoriais, contabilizou-se a prevalência total de anemia encontrada nas crianças de acordo com a classificação de valores de hemoglobina da OMS: 58,5\%.
A diferença entre o grau de anemia definido pela palidez palmar e o grau de anemia classificado conforme 0 exame de sangue realizado em laboratório pode ser decorrente de diversos fatores que interferem na determinação da palidez palmar, tais como raça, temperatura e posição, entre outros. ${ }^{1}$

A Tabela 4 apresenta a prevalência da anemia avaliada pela palidez palmar e o sexo das crianças. 
Tabela 4 Prevalência de anemia ferropriva a partir do método palidez palmar relacionada ao sexo das crianças de 2 meses a 5 anos usuárias de uma ESF do município de Panambi-RS

\begin{tabular}{lcc}
\hline Variáveis & $\mathrm{N}=21$ & $\%$ \\
\hline Prevalência da Anemia pelo Método Palidez Palmar & & \\
& 20 & 48,8 \\
Palidez palmar leve, moderada & 1 & 2,4 \\
Palidez qrave & 20 & 48.8 \\
Pele normal (ausência de anemia) & & \\
Sexo XPalidez Palmar (anemia leve, moderada e grave) & 11 & 52,3 \\
Masculino (palidez Palmar leve) & 10 & 47,6 \\
\hline Feminino (palidez palmar leve e grave) & & \\
\hline
\end{tabular}

Com relação ao sexo, $52,3 \%$ das crianças classificadas pelo método palidez palmar com anemia leve eram do sexo masculino e 47,6\% com palidez palmar leve e moderada eram do sexo feminino. Reforça-se que não existe uma diferença expressiva na prevalência de anemia por sexo, mas a maior ocorrência de anemia em crianças do sexo masculino pode ser desencadeada pela maior velocidade de crescimento nessa faixa etária, ainda que não haja comprovação disso por método estatístico. Salienta-se que este dado pode estar relacionado ao fato de o número total de crianças do estudo ser do sexo masculino.

Tabela 5 Prevalência de anemia relacionada a faixa etária a partir do exame laboratorial, de crianças de 2 meses a 5 anos usuárias de uma ESF do município de Panambi-RS 2009.

\begin{tabular}{|c|c|c|}
\hline Variáveis & $\mathrm{N}=24$ & $\%$ \\
\hline \multicolumn{3}{|l|}{ Prevalência da Anemia pelo Hemograma } \\
\hline Anemia leve (7 e $9,9 \mathrm{~g} / \mathrm{dl})$; & 12 & 50,0 \\
\hline Anemia moderada (<10 g/dl ); & 11 & 45,8 \\
\hline Anemia grave $(? 7 \mathrm{~g} / \mathrm{dl})$ & 1 & 4,1 \\
\hline \multicolumn{3}{|c|}{ Faixa Etária em (meses) $\times$ Hemoglobina $<10 \mathrm{mg}$} \\
\hline 2- 12 meses (leve e moderada) & 15 & 62,5 \\
\hline 13-24 meses (leve, moderada e grave) & 6 & 25,0 \\
\hline 25-36 meses (leve ) & 1 & 4,1 \\
\hline 37-60 meses (leve e moderada) & 2 & 8,3 \\
\hline
\end{tabular}

Quanto à distribuição da anemia pelo resultado da hemoglobina com ponto de corte $<10 \mathrm{mg}$ de hemoglobina, de acordo com a faixa etária, observa-se que, nos menores de 12 meses, a prevalência foi maior e, especificamente, na faixa etária entre 2 e 12 meses, o percentual foi de $62,5 \%$, e, entre 13 a 24 meses, de $25 \%$. Nas crianças acima de 25 meses até 60 meses, a prevalência observada foi de 12,4\%. A prevalência de anemia em menores de 12 meses é um dado estatisticamente considerável nesta população, pois as crianças no primeiro ano de vida tiveram 2,5 vezes mais anemia comparadas às crianças no segundo ano de vida. Esses dados são semelhantes a de outros estudos. A anemia é uma doença que acomete crianças menores de 3 anos. ${ }^{13}$

Um estudo realizado no município de Pelotas - RS com crianças menores de 6 anos sobre a prevalência da anemia revelou um risco bruto de anemia de 12,3 vezes maior no primeiro ano de vida comparado ao de crianças de 5 anos, ${ }^{4}$ sustentando os achados do presente estudo.

Outro estudo, realizado na cidade de Belo Horizonte, mostra que a prevalência da anemia em crianças menores de 24 meses foi o dobro da prevalência de outras faixas etárias e nesse mesmo estudo as crianças entre 24 e 48 meses apresentaram 9,25 e 1,31 vezes mais chance de apresentar anemia. $^{13}$

Estudos semelhantes foram realizados nas cidades de Recife, São Paulo e Tubarão, pois o AIDPI vem se difundindo, principalmente nessas regiões do país, e, apesar dessa estratégia ofertar capacitações para os profissionais aplicarem o método palidez palmar, ainda é necessário avaliar a eficácia e a sensibilidade do teste por sinal clínico.

Uma forma de validar o método clínico de palidez foi a avaliação com dois observadores ou mais, e os resultados apontam para uma baixa concordância na avaliação. ${ }^{11}$ Salienta-se que o fato de os observadores concordarem não significa que a classificação da anemia esteja correta. ${ }^{12}$

Os resultados de estudos comparativos sobre os níveis séricos médios de hemoglobina e palidez graduada não apresentam diferenças estatisticamente significativas entre os graus de palidez atribuídos. ${ }^{11}$

No que se refere à sensibilidade para a palidez palmar em relação ao padrão de diagnóstico de hemoglobina, os estudos têm mostrado baixa sensibilidade. Sendo assim, há maior dificuldade para a avaliação da coloração da palma da mão, e a combinação de mais de uma parte do corpo 
para sustentar a graduação do examinador parece ser um método mais seguro. ${ }^{11}$

Dessa forma, sugere-se que, para a adoção do método de avaliação clínica de palidez, seja necessário aprimorar a técnica, considerando as populações assistidas e suas características sociodemográficas. Por ter caráter subjetivo, necessita de um treinamento intensivo por parte de quem vai aplicar. Além disso, é fácil, simples e sem custo financeiro. ${ }^{11}$

Os estudos citados ressaltam a importância do método palidez palmar da estratégia AIDPI como forma de avaliar anemia severa e salientam a impor tância do conhecimento e da experiência dos examinadores, bem como das condições sociodemográficas das populações assistidas como fator decisivo na aplicação do método.

Embora se reconheça a palidez palmar como método de triagem de anemia, um estudo realizado em Natividade RJ mostrou baixas sensibilidade e especificidade na eficácia e concordância do diagnóstico clínico de anemia pelo exame da palidez palmar comparadas com valores de hemoglobina capilar. A concordância entre os dois métodos foi pior entre as crianças menores de 2 anos de idade e com anemia moderada-grave. ${ }^{10}$

Outro estudo que contribui nessa discussão foi realizado em Recife em um Centro Materno-Infantil com o objetivo de avaliar a validade e reprodutibilidade dos sinais clínicos (palidez palmar e conjuntival) no diagnóstico de anemia em crianças de 6-23 meses de idade. Nele também foi realizado o exame laboratorial para a mensuração da hemoglobina, e foi constatado que a sensibilidade dos sinais clínicos foi melhor para diagnosticar a anemia moderada/grave, especialmente quando a palidez palmar e conjuntival estavam combinadas, sugerindo que a utilização destes não se constitui em um bom instrumento para diagnosticar anemia leve. ${ }^{12}$

0 uso do sinal clínico para avaliar a anemia apresenta baixa sensibilidade e não parece ser um bom indicador para determinar anemia em crianças, considerando que a cor da pele da palma da mão da mãe é comparada à das crianças e mulheres em idade reprodutiva também se constituem em um grupo suscetível à anemia. ${ }^{12}$

Os sinais clínicos apresentam maior sensibilidade quanto maior for o grau de anemia. Dessa forma, em crianças que apresentam anemia moderada, o teste torna-se menos preciso. ${ }^{12}$ No Brasil, a prevalência da anemia é leve, sendo possivelmente decorrente de dificuldade de acesso a alimentos ricos em ferro, e não sugere a adoção do método palidez palmar para avaliação. $^{12}$

Sendo assim, reconhece-se que o método palidez palmar como teste de triagem vem a contribuir para a identificação da anemia em crianças, possibilitando o encaminhamento para investigação, tratamento e prevenção desse problema que acomete mais de $60 \%$ da população infantil especialmente em regiões em que a prevalência da anemia é classificada como grave e associada a exames laboratoriais quando a prevalência da anemia é moderada ou leve.
Por meio do exame clínico da palidez palmar, foi identificado o grau de anemia ferropriva. Pelo fato de a anemia possuir quatro classificações, segundo os critérios da OMS, os tipos moderada e grave foram avaliados em conjunto, decisão tomada previamente pela pesquisadora e pelo médico, ambos observadores e graduadores da anemia.

\section{CONSIDERAÇÕES FINAIS}

Os resultados dessa pesquisa mostram a prevalência de anemia ferropriva superiores a $50 \%$ em crianças de 2 meses a 5 anos de idade. Esses valores são semelhantes aos observados em outras regiões do país. Ressaltam-se os altos índices dessa prevalência, principalmente em crianças com menos de 24 meses de idade.

Um dado observado foi a aproximação entre 0 diagnóstico de anemia pelo exame clínico da palidez palmar e o resultado do exame laboratorial de sangue (hemograma), pelo fato de que as prevalências de anemia encontradas pelos dois métodos foram muito semelhantes. Salienta-se que não foi realizado nenhum teste estatístico para validar o método e a sensibilidade deste.

Identificou-se que o exame da palidez palmar é extremamente eficaz no diagnóstico clínico da anemia ferropriva nas crianças portadoras de anemia grave, pois a palidez palmar pôde ser nitidamente observada. Nesse contexto, mesmo reconhecendo a eficácia do exame da palidez palmar e do hemograma separadamente, é importante lembrar que o exame clínico e o exame laboratorial, quando usados em conjunto, podem fornecer um diagnóstico muito mais preciso e confiável.

A alta proporção de crianças anêmicas observadas neste estudo indica a necessidade de salientar, nos programas de pré-natal e puericultura do Brasil, medidas de promoção, prevenção e controle dessa doença com mais eficácia, pois nota-se que, apesar de existirem ações de avaliação e medidas, a sua prevalência continua aumentando de forma descontrolada. Os dados também confirmam que as condições sociodemográficas, como sexo, idade, escolaridade e o grau de instrução, são fatores que interferem diretamente na incidência da anemia ferropriva em crianças. Se forem modificados, esses aspectos poderão determinar menores coeficientes da doença.

Apesar de a Agência Nacional de Vigilância Sanitária (ANVISA) ter tornado obrigatória a fortificação de farinhas de trigo e milho com ferro e ácido fólico, e também o Ministério da Saúde ter apresentado medidas precisas para o tratamento medicamentoso da anemia através do Programa Nacional de Suplementação de Ferro pela Portaria n 730/GM, são necessárias outras medidas para reduzir essa prevalência. Uma delas seria a educação em saúde, de maneira a proporcionar a essa população maiores informações e orientações a respeito dessa patologia.

Dentre os limites do estudo, salienta-se a amostra, pequena, porém suficiente para validar estudos já realizados com populações maiores. Sugere-se a continuação deste estudo 
com a ampliação da amostra para maior precisão, e também a realização de testes estatísticos que validem a sensibilidade do método.

Este estudo mostrou a importância da estratégia do AIDPI como modelo de identificação da anemia em um contexto que apresenta características sociodemográficas específicas. Também permitiu que essa metodologia fosse experimentada com a participação de uma equipe multiprofissional (médico, enfermeiro, técnicos de enfermagem e acadêmica de enfermagem), fortalecendo a pesquisa como forma de aprendizado, produção de conhecimento e intervenção.

A ampliação da estratégia AIDPI em unidades de saúde da família está avançando no Brasil, porém, com dificuldades de ordem técnica assistencial apresentada por parte dos profissionais de saúde e organizacional apresentada por parte dos gestores. Para enfrentar essas dificuldades, o Ministério da Saúde tem ofertado capacitações para dar segurança aos profissionais que vão executar a estratégia AIDPI. A participação da enfermagem nesta proposta pode se dar em vários momentos na avaliação da saúde da criança compartilhada com o médico.

A classificação da anemia e a avaliação da alimentação da criança para o aconselhamento da mãe ao uso de suplementação de ferro e/ou tratamento quando necessário, e o devido acompanhamento de acordo com as recomendações propostas pelo Ministério da Saúde, são ações, entre outras, que podem ser incluídas na prática assistencial da enfermagem. Nesse aspecto, proporcionar à criança um cuidado ancorado na metodologia AIDPI para mensurar a anemia em crianças poderá complementar ações que integrem a Agenda de Compromissos para a Saúde Integral da Criança e Redução da Mortalidade Infantil.

\section{REFERÊNCIAS}

1 Organização Pan-Americana da Saúde-OMS. AIDPI para o ensino médico: manual de apoio. Brasília(DF); 2004.

2 Kraemer K, Zimmermann MB. Nutricional anemia. Basel, Switzerland: Sight and Life Press. 2007. [citado 2007 jul 24]. Disponível em: <http:// www.who.int/nutrition/publications/>.

3 Torres MAA. Anemia carencial ferropriva. In: Nóbrega FJ. Distúrbios da nutrição na infância e na adolescência. Rio de Janeiro: Revinter; 2007. p 355-59.

4 Santos I, César JA, Minten G, Valle N, Neumann NA, Cercato E. Prevalência e fatores associados à ocorrência de anemia entre menores de seis anos de idade em Pelotas, RS. Rev Bras Epidemiol. [periódico on-line]. 2004 dez [citado 2010 out 02]; 7(4): 403-415. Disponivel em: http:// www.scielosp.org/scielo.php?script=sci_arttext\&pid=S1415$790 \times 2004000400004 \& \mathrm{lng}=$ pt. doi: 10.1590/S1415$790 \times 2004000400004$
5 Bueno MB, Selem SSC, Arêas JA, Gomes FRM. Prevalência e fatores associados à anemia entre crianças atendidas em creches públicas de São Paulo. Rev Bras Epidemiol. [periódico on-line]. 2006 dez [citado 2010 out 02] ; 9(4): 462-70. Disponível em: http://www.scielosp.org/ scielo.php?script =sci_arttext $\alpha$ pid $=$ S 1415 790X2006000400007\&lng=pt. doi: 10.1590/S1415$790 \times 2006000400007$.

6 Silveira SV, Albuquerque LC, Rocha EJM, Martins MCV. Fatores de risco associados a anemia ferropriva em crianças de 12 a 36 meses de creches públicas de Fortaleza. Rev Pediatr. 2008 jul/dez; 9 (2): 70-6.

7 World Health Organization- WHO. Iron deficiency anaemia. assessment, prevention and control. A guide for programme managers. Geneva; 2001. 8 Ministério da Saúde(BR). AIDPI: Atenção Integral às doenças Prevalentes na Infância. Manual de Capacitação 1. Brasília (DF); 2002.

9 Ministério da Saúde(BR) Atenção Integral as Doenças Prevalentes na

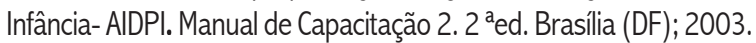

10 Rezende LRC, Lamounier JAL, Magalhães WLR, Bragança CR, Rezende PIRC, Bohrer FJM, et al. Eficácia do exame clínico para o diagnóstico de anemia na criança pela palidez palmar e dosagem de hemoglobina. Pediatria. 2009; 31(3): 161-69.

11 Spinelli MGN, Souza JMP, Souza SB, Sesoko EH. Confiabilidade e validade da palidez palmar e de conjuntivas como triagem de anemia. Rev Saude Publica. [periódico on-line]. 2003 ago [citado 2010 out 02] ; 37(4): 404-08. Disponível em: http://www.scielosp.org/ scielo.php?script=sci_arttext $\&$ pid=S0034$89102003000400003 \& \operatorname{lng}=$ pt. doi: 10.1590/S003489102003000400003

12 Leal LP, Osório MM. Validação e reprodutibilidade de sinais clínicos no diagnóstico de anemia em crianças. Cad Saude Publica. [periódico na Internet]. 2005 abr [citado 2010 out 02]; 21(2): 565-72. Disponível em: http://www.scielosp.org/scielo.php?script=sci_arttext\&pid=S0102$311 \times 2005000200023 \& l n g=p t . \quad$ doi: $10.1590 /$ S0102$311 \times 2005000200023$

13. Rocha DS, Lamounier JA, Capanema FD, Franceschini SCC, Norton RC, Costa ABP. et al . Estado nutricional e prevalência de anemia em crianças que freqüentam creches em Belo Horizonte, Minas Gerais. Rev Paul Pediatr. [periódico on-line]. 2008 mar [cited 2010 out 02]; 26(1): 6-13. Disponível em: http://www.scielo.br/ scielo.php?script=sci_arttext\&pid=s010305822008000100002\&lng=en. doi: 10.1590/S010305822008000100002.

14 Osório MM. Fatores determinantes da anemia em crianças. J Pediatr. [periódico on-line]. 2002 [cited 2010 out 02]; 78(4): 269-78. Disponível em: http://www.scielo.br/scielo.php?script=sci_arttext\&pid=S002175572002000400005\&lng=en. doi: 10.1590/S002175572002000400005 .

15 Brito LL, Barreto ML, Silva RCR, Assis AMO, Reis MG, Parraga I. et al . Fatores de risco para anemia por deficiência de ferro em crianças e adolescentes parasitados por helmintos intestinais. Rev Panam Salud Publica. [periódico on-line]. 2003 dez [citado 2010 out 02]; 14(6): 
422-431. Disponível em: http://www.scielosp.org/ scielo.php?script=sci_arttext $\&$ pid $=S 1020$ 49892003001100007\&lng=en. doi: 10.1590/S102049892003001100007.

16 Pedroso MLR, Motta. MGC. Vulnerabilidades socioeconômicas e 0 cotidiano da assistência de enfermagem pediátrica: relato de enfermeiras. Esc Anna Nery. 2010; 14(2): 293-300.

17 Oliveira GIC, Resende LM, Matos SP, Soares EM. Alimentação e suplementação de ferro em uma população de lactentes carentes. Pediatria. 2006; 28(1): 18-25.

18 Jordão RE, Bernardi JLD, Barros Filho AA. Prevalência de anemia ferropriva no Brasil: uma revisão sistemática. Rev Paul Pediatr. [periódico on-line]. 2009 mar [citado 2010 out 02]; 27(1): 90-98. Available from: http://www.scielo.br/scielo.php?script=sci_arttext\&pid=S010305822009000100014\&lng=en. doi: 10.1590/S010305822009000100014. 